Network Working Group

Request for Comments: 4523

Obsoletes: 2252, 2256, 2587

Category: Standards Track
K. Zeilenga

OpenLDAP Foundation

June 2006

Lightweight Directory Access Protocol (LDAP)
Schema Definitions for X.509 Certificates

Status of This Memo

This document specifies an Internet standards track protocol for the Internet community, and requests discussion and suggestions for improvements. Please refer to the current edition of the "Internet Official Protocol Standards" (STD 1) for the standardization state and status of this protocol. Distribution of this memo is unlimited.

Copyright Notice

Copyright (C) The Internet Society (2006).

Abstract

This document describes schema for representing X.509 certificates, X.521 security information, and related elements in directories accessible using the Lightweight Directory Access Protocol (LDAP). The LDAP definitions for these X.509 and X.521 schema elements replace those provided in RFCs 2252 and 2256.

1. Introduction

This document provides LDAP [RFC4510] schema definitions [RFC4512] for a subset of elements specified in X.509 [X.509] and X.521 [X.521], including attribute types for certificates, cross certificate pairs, and certificate revocation lists; matching rules to be used with these attribute types; and related object classes. LDAP syntax definitions are also provided for associated assertion and attribute values.

As the semantics of these elements are as defined in X.509 and X.521, knowledge of X.509 and X.521 is necessary to make use of the LDAP schema definitions provided herein.

This document, together with [RFC4510], obsoletes RFCs 2252 and 2256 in their entirety. The changes (in this document) made since RFC 2252 and RFC 2256 include:

$$
\text { - addition of pkiUser, pkiCA, and deltaCRL classes; }
$$


- update of attribute types to include equality matching rules in accordance with their $\mathrm{X} .500$ specifications;

- addition of certificate, certificate pair, certificate list, and algorithm identifier matching rules; and

- addition of LDAP syntax for assertion syntaxes for these matching rules.

This document obsoletes RFC 2587. The X.509 schema descriptions for LDAPv2 [RFC1777] are Historic, as is LDAPv2 [RFC3494].

The key words "MUST", "MUST NOT", "REQUIRED", "SHALL", "SHALL NOT", "SHOULD", "SHOULD NOT", "RECOMMENDED", "MAY", and "OPTIONAL" in this document are to be interpreted as described in BCP 14 [RFC2119].

Schema definitions are provided using LDAP description formats [RFC4512]. Definitions provided here are formatted (line wrapped) for readability.

2. Syntaxes

This section describes various syntaxes used in LDAP to transfer certificates and related data types.

\subsection{Certificate}

$$
\text { ( 1.3.6.1.4.1.1466.115.121.1.8 DESC 'X.509 Certificate' ) }
$$

A value of this syntax is an X.509 Certificate [X.509, clause 7].

Due to changes made to the definition of a Certificate through time, no LDAP-specific encoding is defined for this syntax. Values of this syntax SHOULD be encoded using Distinguished Encoding Rules (DER) [X.690] and MUST only be transferred using the ; binary transfer option [RFC4522]; that is, by requesting and returning values using attribute descriptions such as "userCertificate; binary".

As values of this syntax contain digitally signed data, values of this syntax and the form of each value MUST be preserved as presented.

\subsection{Certificatelist}

$$
\text { ( 1.3.6.1.4.1.1466.115.121.1.9 DESC 'X.509 Certificate List' ) }
$$

A value of this syntax is an X.509 Certificatelist [X.509, clause 7.3]. 
Due to changes made to the definition of a Certificatelist through time, no LDAP-specific encoding is defined for this syntax. Values of this syntax SHOULD be encoded using DER [X.690] and MUST only be transferred using the ; binary transfer option [RFC4522]; that is, by requesting and returning values using attribute descriptions such as "certificateRevocationList; binary".

As values of this syntax contain digitally signed data, values of this syntax and the form of each value MUST be preserved as presented.

\subsection{CertificatePair}

$$
\text { ( 1.3.6.1.4.1.1466.115.121.1.10 DESC 'X.509 Certificate Pair' ) }
$$

A value of this syntax is an X.509 CertificatePair [X.509, clause $11.2 .3]$.

Due to changes made to the definition of an X.509 CertificatePair through time, no LDAP-specific encoding is defined for this syntax. Values of this syntax SHOULD be encoded using DER [X.690] and MUST only be transferred using the ; binary transfer option [RFC4522]; that is, by requesting and returning values using attribute descriptions such as "crossCertificatePair;binary".

As values of this syntax contain digitally signed data, values of this syntax and the form of each value MUST be preserved as presented.

\subsection{SupportedAlgorithm}

( 1.3 .6 .1 .4 .1 .1466 .115 .121 .1 .49

DESC 'X.509 Supported Algorithm' )

A value of this syntax is an X.509 SupportedAlgorithm [X.509, clause $11.2 .7]$.

Due to changes made to the definition of an X.509 SupportedAlgorithm through time, no LDAP-specific encoding is defined for this syntax. Values of this syntax SHOULD be encoded using DER [X.690] and MUST only be transferred using the ; binary transfer option [RFC4522]; that is, by requesting and returning values using attribute descriptions such as "supportedAlgorithms; binary".

As values of this syntax contain digitally signed data, values of this syntax and the form of the value MUST be preserved as presented. 


\title{
2.5. CertificateExactAssertion
}

$$
\text { ( 1.3.6.1.1.15.1 DESC 'X.509 Certificate Exact Assertion' ) }
$$

A value of this syntax is an X.509 CertificateExactAssertion [X.509, clause 11.3.1]. Values of this syntax MUST be encoded using the Generic String Encoding Rules (GSER) [RFC3641]. Appendix A.1 provides an equivalent Augmented Backus-Naur Form (ABNF) [RFC4234] grammar for this syntax.

2.6. CertificateAssertion

$$
\text { ( 1.3.6.1.1.15.2 DESC 'X.509 Certificate Assertion' ) }
$$

A value of this syntax is an X.509 CertificateAssertion [X.509, clause 11.3.2]. Values of this syntax MUST be encoded using GSER [RFC3641]. Appendix A.2 provides an equivalent ABNF [RFC4234] grammar for this syntax.

\subsection{CertificatePairExactAssertion}

$$
\begin{aligned}
& \text { ( } 1.3 .6 .1 .1 .15 .3 \\
& \text { DESC 'X.509 Certificate Pair Exact Assertion' ) }
\end{aligned}
$$

A value of this syntax is an X.509 CertificatePairExactAssertion [X.509, clause 11.3.3]. Values of this syntax MUST be encoded using GSER [RFC3641]. Appendix A.3 provides an equivalent ABNF [RFC4234] grammar for this syntax.

\subsection{CertificatePairAssertion}

$$
\text { ( 1.3.6.1.1.15.4 DESC 'X.509 Certificate Pair Assertion' ) }
$$

A value of this syntax is an X.509 CertificatePairAssertion [X.509, clause 11.3.4]. Values of this syntax MUST be encoded using GSER [RFC3641]. Appendix A.4 provides an equivalent ABNF [RFC4234] grammar for this syntax.

\subsection{CertificatelistExactAssertion}

\author{
$(1.3 \cdot 6 \cdot 1 \cdot 1 \cdot 15 \cdot 5$ \\ DESC 'X.509 Certificate List Exact Assertion' )
}

A value of this syntax is an X.509 CertificatelistExactAssertion [X.509, clause 11.3.5]. Values of this syntax MUST be encoded using GSER [RFC3641]. Appendix A.5 provides an equivalent ABNF grammar for this syntax. 


\subsection{CertificatelistAssertion}

$$
\text { ( 1.3.6.1.1.15.6 DESC 'X.509 Certificate List Assertion' ) }
$$

A value of this syntax is an X.509 CertificatelistAssertion [X.509, clause 11.3.6]. Values of this syntax MUST be encoded using GSER [RFC3641]. Appendix A.6 provides an equivalent ABNF [RFC4234] grammar for this syntax.

2.11. AlgorithmIdentifier

$$
\text { ( 1.3.6.1.1.15.7 DESC 'X.509 Algorithm Identifier' ) }
$$

A value of this syntax is an X.509 AlgorithmIdentifier [X.509, Clause 7]. Values of this syntax MUST be encoded using GSER [RFC3641].

Appendix A.7 provides an equivalent ABNF [RFC4234] grammar for this syntax.

3. Matching Rules

This section introduces a set of certificate and related matching rules for use in LDAP. These rules are intended to act in accordance with their X.500 counterparts.

\section{1. certificateExactMatch}

The certificateExactMatch matching rule compares the presented certificate exact assertion value with an attribute value of the certificate syntax as described in clause 11.3.1 of [X.509].

\section{( 2.5.13.34 NAME 'certificateExactMatch' DESC 'X.509 Certificate Exact Match' SYNTAX 1.3.6.1.1.15.1)}

\section{2. certificateMatch}

The certificateMatch matching rule compares the presented certificate assertion value with an attribute value of the certificate syntax as described in clause 11.3.2 of [X.509].

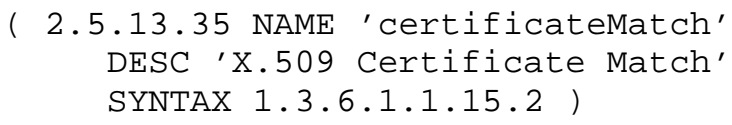




\section{3. certificatePairExactMatch}

The certificatePairExactMatch matching rule compares the presented certificate pair exact assertion value with an attribute value of the certificate pair syntax as described in clause 11.3.3 of [X.509].

\section{( 2.5.13.36 NAME 'certificatePairExactMatch' DESC 'X.509 Certificate Pair Exact Match' SYNTAX 1.3.6.1.1.15.3)}

3.4. certificatePairMatch

The certificatePairMatch matching rule compares the presented certificate pair assertion value with an attribute value of the certificate pair syntax as described in clause 11.3.4 of [X.509].

\section{( 2.5.13.37 NAME ' certificatePairMatch' DESC 'X.509 Certificate Pair Match' SYNTAX 1.3.6.1.1.15.4)}

\section{5. certificatelistexactMatch}

The certificateListExactMatch matching rule compares the presented certificate list exact assertion value with an attribute value of the certificate pair syntax as described in clause 11.3 .5 of [X.509].

\section{( 2.5.13.38 NAME 'certificateListExactMatch' DESC 'X.509 Certificate List Exact Match' SYNTAX 1.3.6.1.1.15.5)}

\section{6. certificatelistMatch}

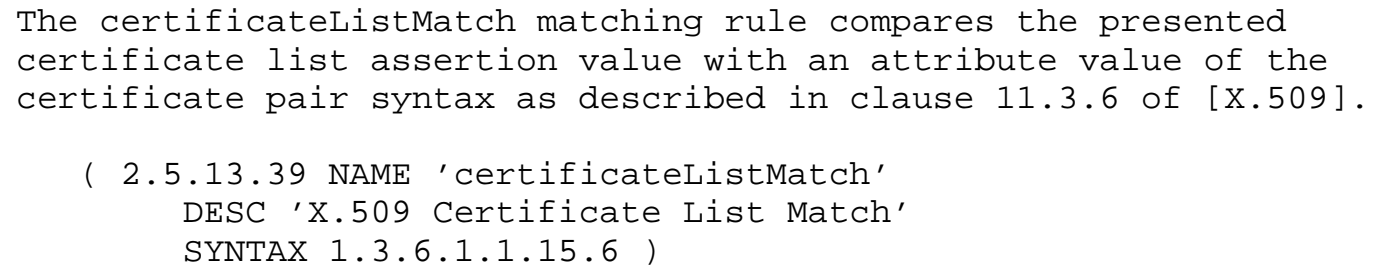




\section{7. algorithmIdentifierMatch}

The algorithmIdentifierMatch mating rule compares a presented algorithm identifier with an attribute value of the supported algorithm as described in clause 11.3 .7 of [X.509].

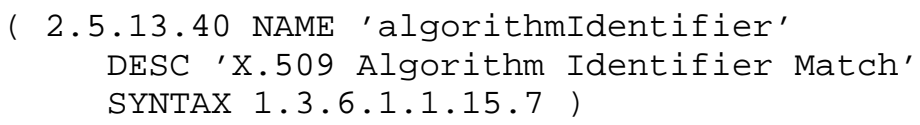

4. Attribute Types

This section details a set of certificate and related attribute types for use in LDAP.

\section{1. userCertificate}

The userCertificate attribute holds the X.509 certificates issued to the user by one or more certificate authorities, as discussed in clause 11.2.1 of [X.509].

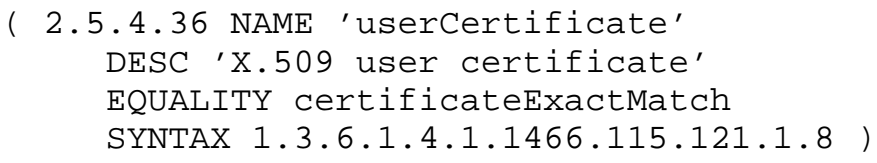

As required by this attribute type's syntax, values of this attribute are requested and transferred using the attribute description "userCertificate; binary".

\section{2. cACertificate}

The cACertificate attribute holds the X.509 certificates issued to the certificate authority (CA), as discussed in clause 11.2 .2 of [X.509].

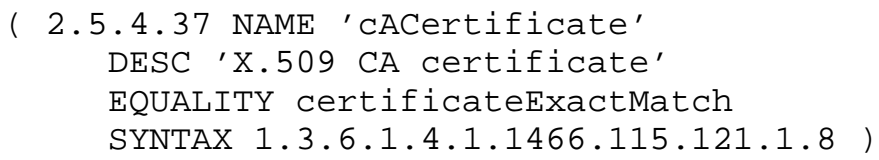

As required by this attribute type's syntax, values of this attribute are requested and transferred using the attribute description "cACertificate; binary". 


\section{3. crosscertificatePair}

The crossCertificatePair attribute holds an X.509 certificate pair, as discussed in clause 11.2.3 of [X.509].

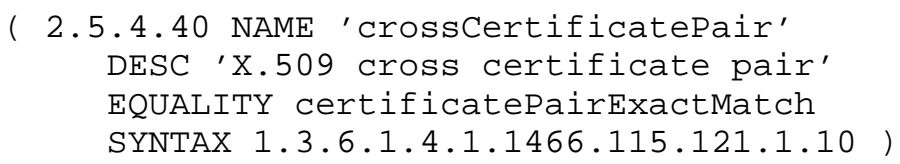

As required by this attribute type's syntax, values of this attribute are requested and transferred using the attribute description "crosscertificatePair;binary".

4.4. certificateRevocationList

The certificateRevocationList attribute holds certificate lists, as discussed in 11.2.4 of [X.509].

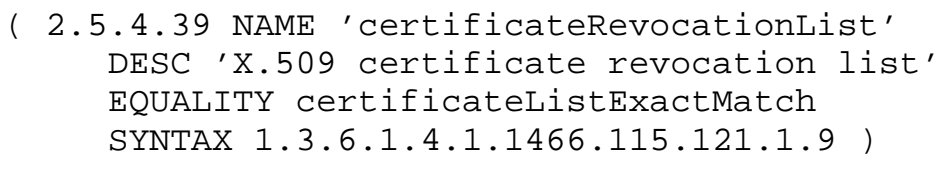

As required by this attribute type's syntax, values of this attribute are requested and transferred using the attribute description

"certificateRevocationList; binary".

4.5. authorityRevocationList

The authorityRevocationList attribute holds certificate lists, as discussed in 11.2.5 of [X.509].

$$
\begin{gathered}
\text { ( 2.5.4.38 NAME ' authorityRevocationList' } \\
\text { DESC 'X.509 authority revocation list' } \\
\text { EQUALITY certificateListExactMatch } \\
\text { SYNTAX 1.3.6.1.4.1.1466.115.121.1.9 ) }
\end{gathered}
$$

As required by this attribute type's syntax, values of this attribute are requested and transferred using the attribute description "authorityRevocationList; binary" . 
4.6. deltaRevocationList

The deltaRevocationList attribute holds certificate lists, as discussed in 11.2.6 of [X.509].

\section{( 2.5.4.53 NAME 'deltaRevocationList' DESC 'X.509 delta revocation list' EQUALITY certificateListExactMatch SYNTAX 1.3.6.1.4.1.1466.115.121.1.9)}

As required by this attribute type's syntax, values of this attribute MUST be requested and transferred using the attribute description "deltaRevocationList; binary".

4.7. supportedAlgorithms

The supportedAlgorithms attribute holds supported algorithms, as discussed in 11.2.7 of [X.509].

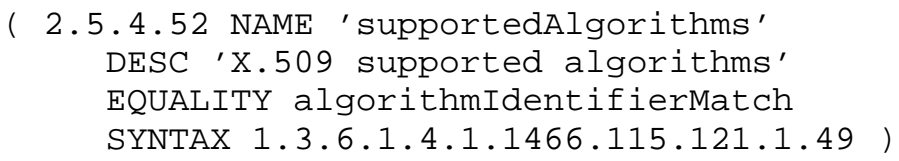

As required by this attribute type's syntax, values of this attribute MUST be requested and transferred using the attribute description "supportedAlgorithms; binary".

5. Object Classes

This section details a set of certificate-related object classes for use in LDAP.

\section{1. pkiuser}

This object class is used in augment entries for objects that may be subject to certificates, as defined in clause 11.1 .1 of [X.509].

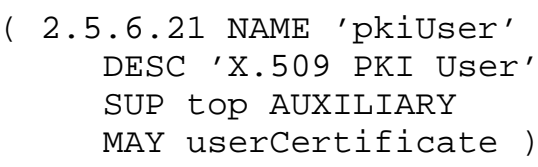




\section{2. pkiCA}

This object class is used to augment entries for objects that act as certificate authorities, as defined in clause 11.1.2 of [X.509]

( 2.5.6.22 NAME 'pkiCA'

DESC 'X.509 PKI Certificate Authority'

SUP top AUXILIARY

MAY ( CACertificate \$ certificateRevocationList \$ authorityRevocationList \$ crossCertificatePair ) )

5.3. CRLDistributionPoint

This class is used to represent objects that act as CRL distribution points, as discussed in clause 11.1 .3 of [X.509].

( 2.5.6.19 NAME 'CRLDistributionPoint' DESC 'X.509 CRL distribution point' SUP tOP STRUCTURAL

MUST $\mathrm{cn}$

MAY ( certificateRevocationList \$ authorityRevocationList \$ deltaRevocationList ) )

5.4. deltacRL

The deltaCRL object class is used to augment entries to hold delta revocation lists, as discussed in clause 11.1.4 of [X.509].

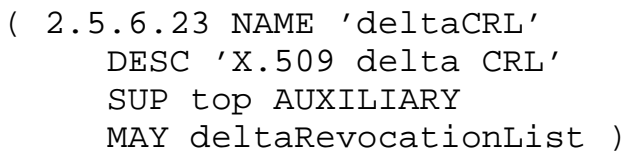

5.5. strongAuthenticationUser

This object class is used to augment entries for objects participating in certificate-based authentication, as defined in clause 6.15 of [X.521]. This object class is deprecated in favor of pkiUser.

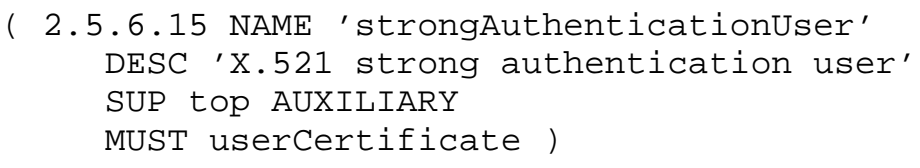




\section{6. userSecurityInformation}

This object class is used to augment entries with needed additional associated security information, as defined in clause 6.16 of [X.521].

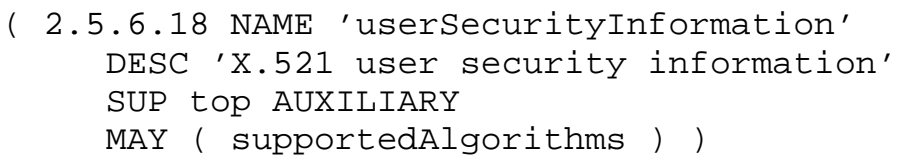

5.7. certificationAuthority

This object class is used to augment entries for objects that act as certificate authorities, as defined in clause 6.17 of [X.521]. This object class is deprecated in favor of pkicA.

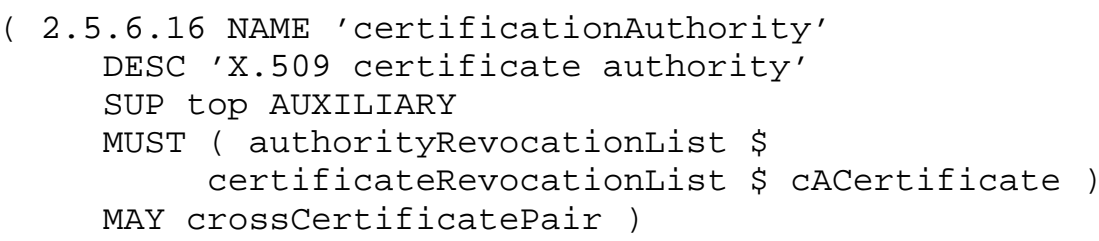

5.8. certificationAuthority-V2

This object class is used to augment entries for objects that act as certificate authorities, as defined in clause 6.18 of [X.521]. This object class is deprecated in favor of pkicA.

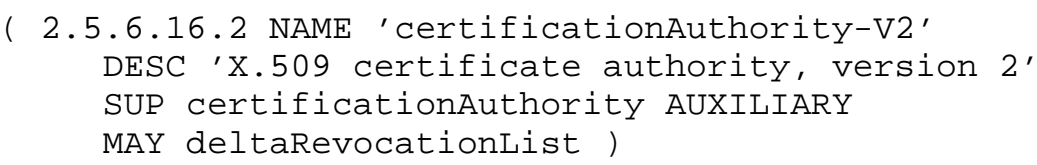

6. Security Considerations

General certificate considerations [RFC3280] apply to LDAP-aware certificate applications. General LDAP security considerations [RFC4510] apply as well.

While elements of certificate information are commonly signed, these signatures only protect the integrity of the signed information. In the absence of data integrity protections in LDAP (or lower layer, e.g., IPsec), a server is not assured that client certificate request (or other request) was unaltered in transit. Likewise, a client cannot be assured that the results of the query were unaltered in 
transit. Hence, it is generally recommended that implementations make use of authentication and data integrity services in LDAP

[RFC4513] [RFC4511].

\section{IANA Considerations}

\subsection{Object Identifier Registration}

The IANA has registered an LDAP Object Identifier [RFC4520] for use in this technical specification.

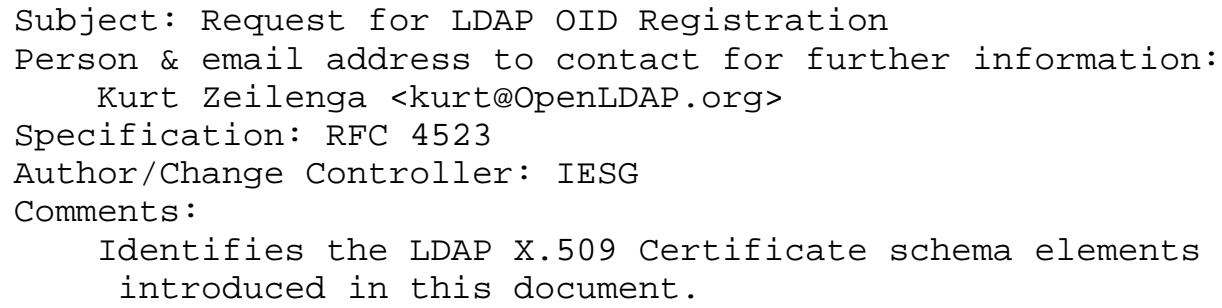

7.2. Descriptor Registration

The IANA has updated the LDAP Descriptor registry [RFC44520] as indicated below.

Subject: Request for LDAP Descriptor Registration 


\begin{tabular}{|c|c|}
\hline deltaCRL & 02.5 .6 .23 \\
\hline deltaRevocationList & A 2.5 .4 .53 \\
\hline pkiCA & 02.5 .6 .22 \\
\hline pkiUser & 2.5 .6 .21 \\
\hline strongAuthenticationUser & 02.5 .6 .15 \\
\hline supportedAlgorithms & A 2.5 .4 .52 \\
\hline userCertificate & A 2.5 .4 .36 \\
\hline serSecurityInformation & 2.5 .6 .18 \\
\hline
\end{tabular}

* Updates previous registration

8. Acknowledgements

This document is based on X.509, a product of the ITU-T. A number of LDAP schema definitions were based on those found in RFCs 2252 and 2256, both products of the IETF ASID WG. The ABNF productions in Appendix A were provided by Steven Legg. Additional material was borrowed from prior works by David Chadwick and Steven Legg to refine the LDAP X.509 schema.

9. References

9.1. Normative References

[RFC2119] Bradner, S., "Key words for use in RFCs to Indicate Requirement Levels", BCP 14, RFC 2119, March 1997.

[RFC3641] Legg, S., "Generic String Encoding Rules (GSER) for ASN.1 Types", RFC 3641, October 2003.

[RFC4510] Zeilenga, K., Ed., "Lightweight Directory Access Protocol (LDAP): Technical Specification Road Map", RFC 4510, June 2006 .

[RFC4512] Zeilenga, K., "Lightweight Directory Access Protocol (LDAP): Directory Information Models", RFC 4512, June 2006 .

[RFC4522] Legg, S., "Lightweight Directory Access Protocol (LDAP): The Binary Encoding Option", RFC 4522, June 2006.

[X.509] International Telecommunication Union - Telecommunication Standardization Sector, "The Directory: Authentication Framework", X.509(2000). 
[X.521] International Telecommunication Union - Telecommunication Standardization Sector, "The Directory: Selected Object Classes", X.521(2000).

[X.690] International Telecommunication Union - Telecommunication Standardization Sector, "Specification of ASN.1 encoding rules: Basic Encoding Rules (BER), Canonical Encoding Rules (CER), and Distinguished Encoding Rules (DER)", $\mathrm{X} .690(2002)$ (also ISO/IEC 8825-1:2002).

9.2. Informative References

[RFC1777] Yeong, W., Howes, T., and S. Kille, "Lightweight Directory Access Protocol", RFC 1777, March 1995.

[RFC2156] Kille, S., "MIXER (Mime Internet X.400 Enhanced Relay): Mapping between X.400 and RFC 822/MIME", RFC 2156, January 1998.

[RFC3280] Housley, R., Polk, W., Ford, W., and D. Solo, "Internet X.509 Public Key Infrastructure Certificate and Certificate Revocation List (CRL) Profile", RFC 3280, April 2002 .

[RFC3494] Zeilenga, K., "Lightweight Directory Access Protocol version 2 (LDAPv2) to Historic Status", RFC 3494, March 2003 .

[RFC3642] Legg, S., "Common Elements of Generic String Encoding Rules (GSER) Encodings", RFC 3642, October 2003.

[RFC4234] Crocker, D. and P. Overell, "Augmented BNF for Syntax Specifications: ABNE", RFC 4234, October 2005.

[RFC4511] Sermersheim, J., Ed., "Lightweight Directory Access Protocol (LDAP): The Protocol", RFC 4511, June 2006.

[RFC4513] Harrison, R. Ed., "Lightweight Directory Access Protocol (LDAP): Authentication Methods and Security Mechanisms", RFC 4513, June 2006.

[RFC4520] Zeilenga, K., "Internet Assigned Numbers Authority (IANA) Considerations for the Lightweight Directory Access Protocol (LDAP)", BCP 64, RFC 4520, June 2006. 
Appendix A.

This appendix is informative.

This appendix provides ABNF [RFC4234] grammars for GSER-based [RFC3641] LDAP-specific encodings specified in this document. These grammars where produced using, and relying on, Common Elements for GSER Encodings [RFC3642].

A.1. CertificateExactAssertion

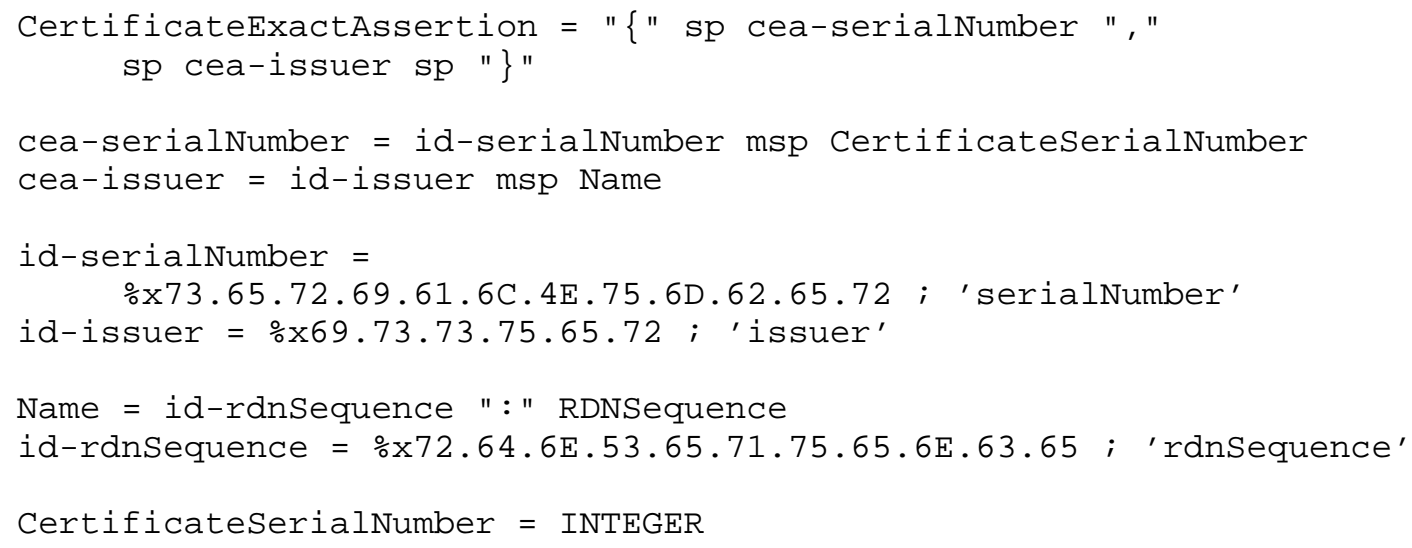




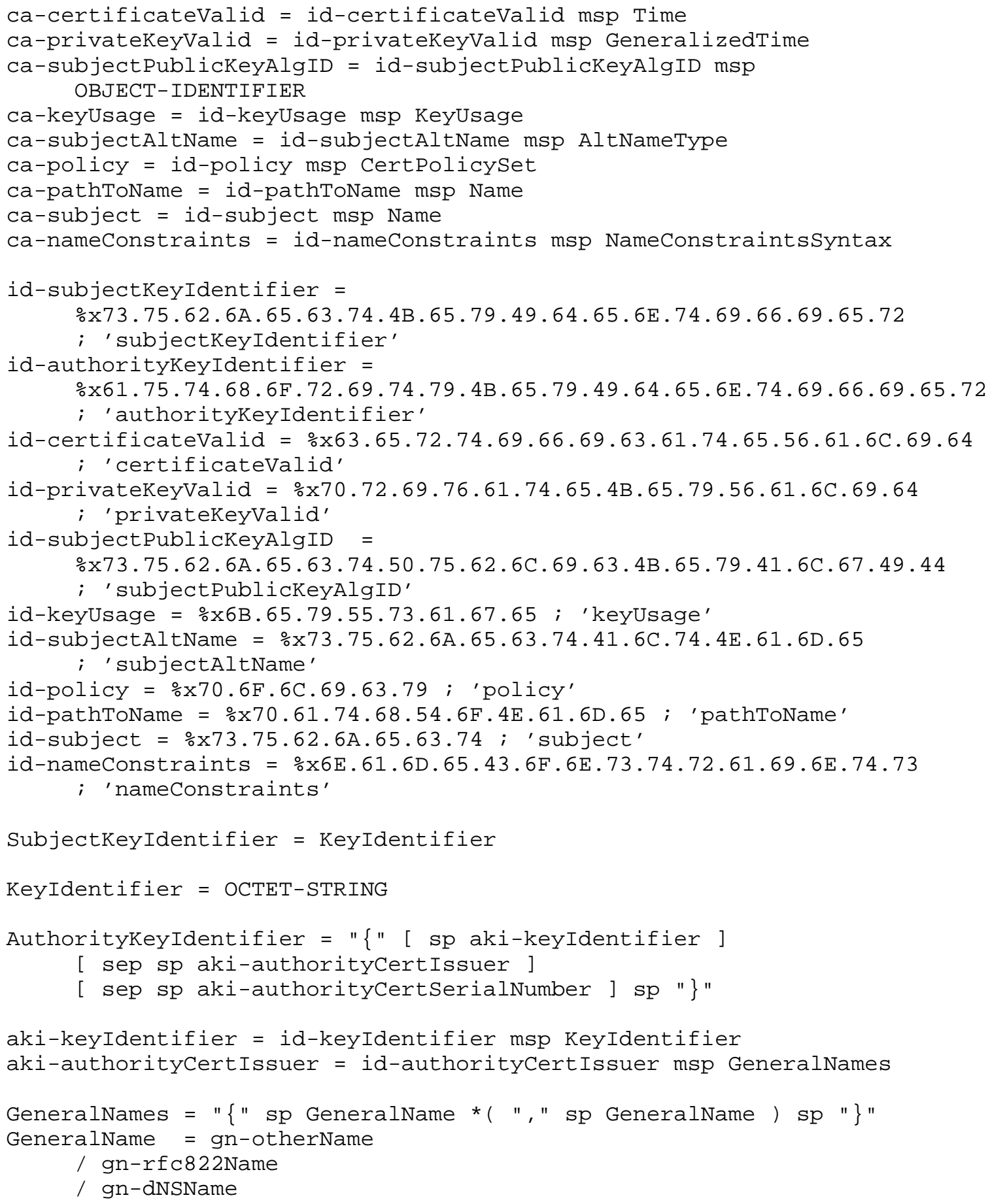




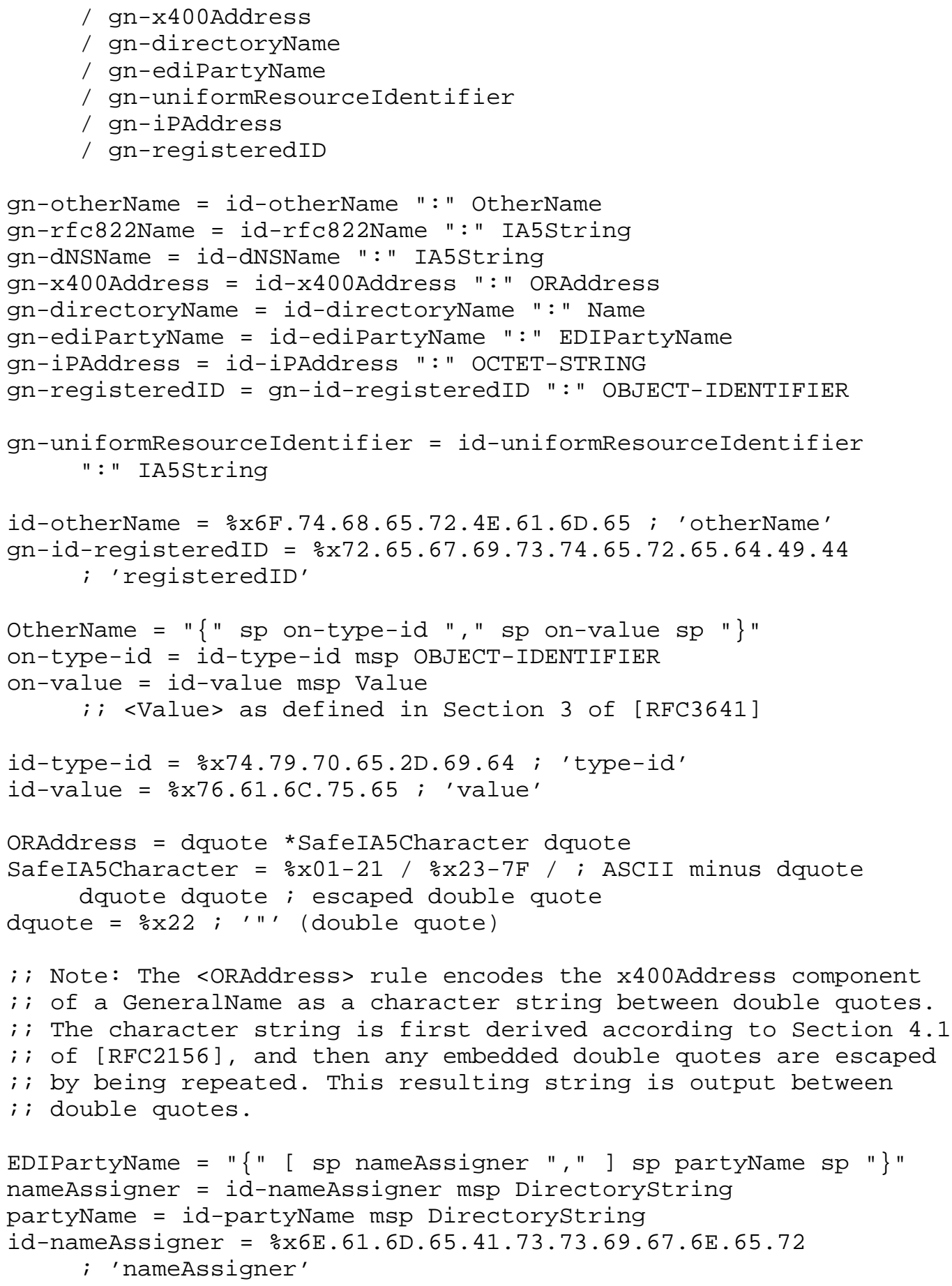




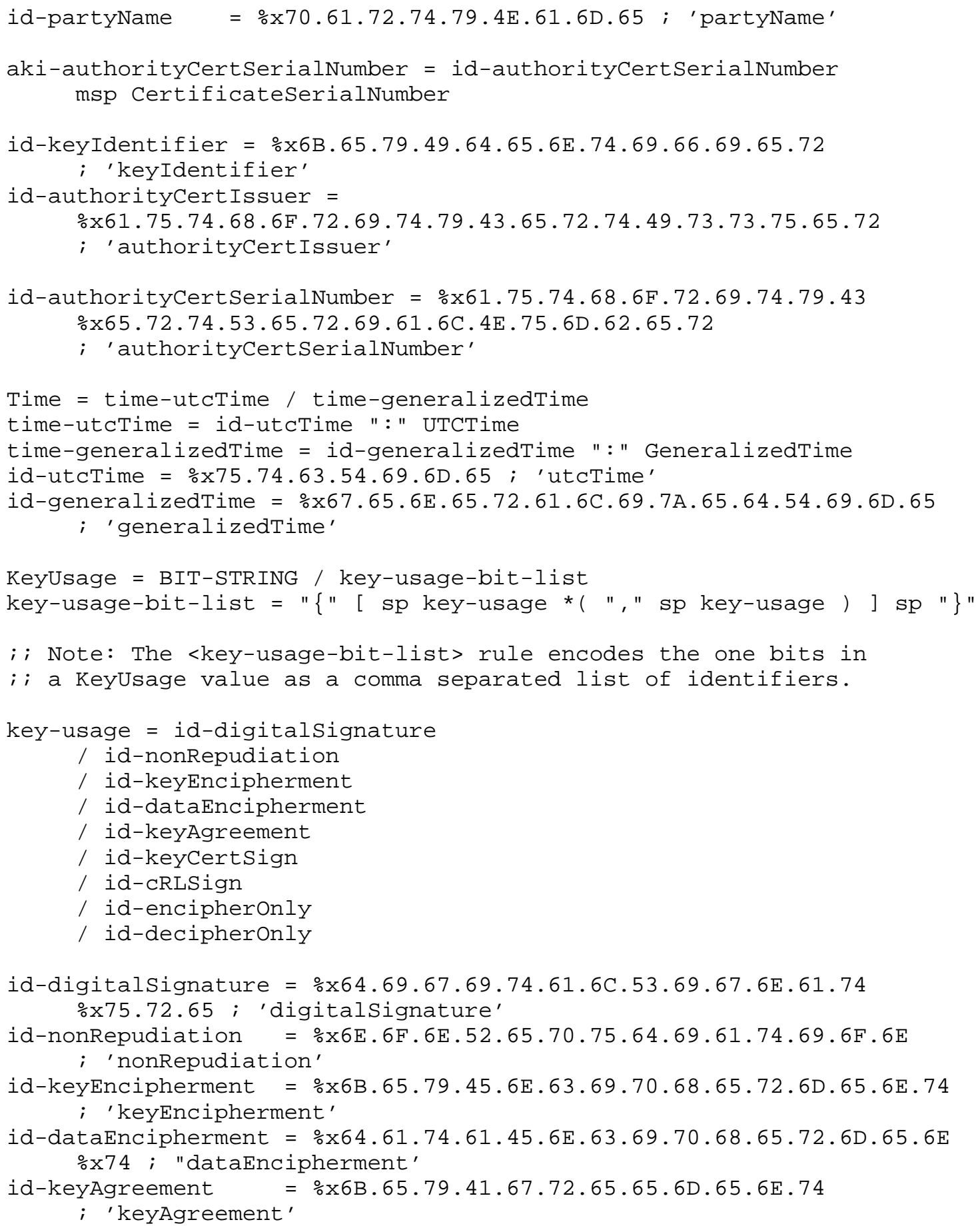




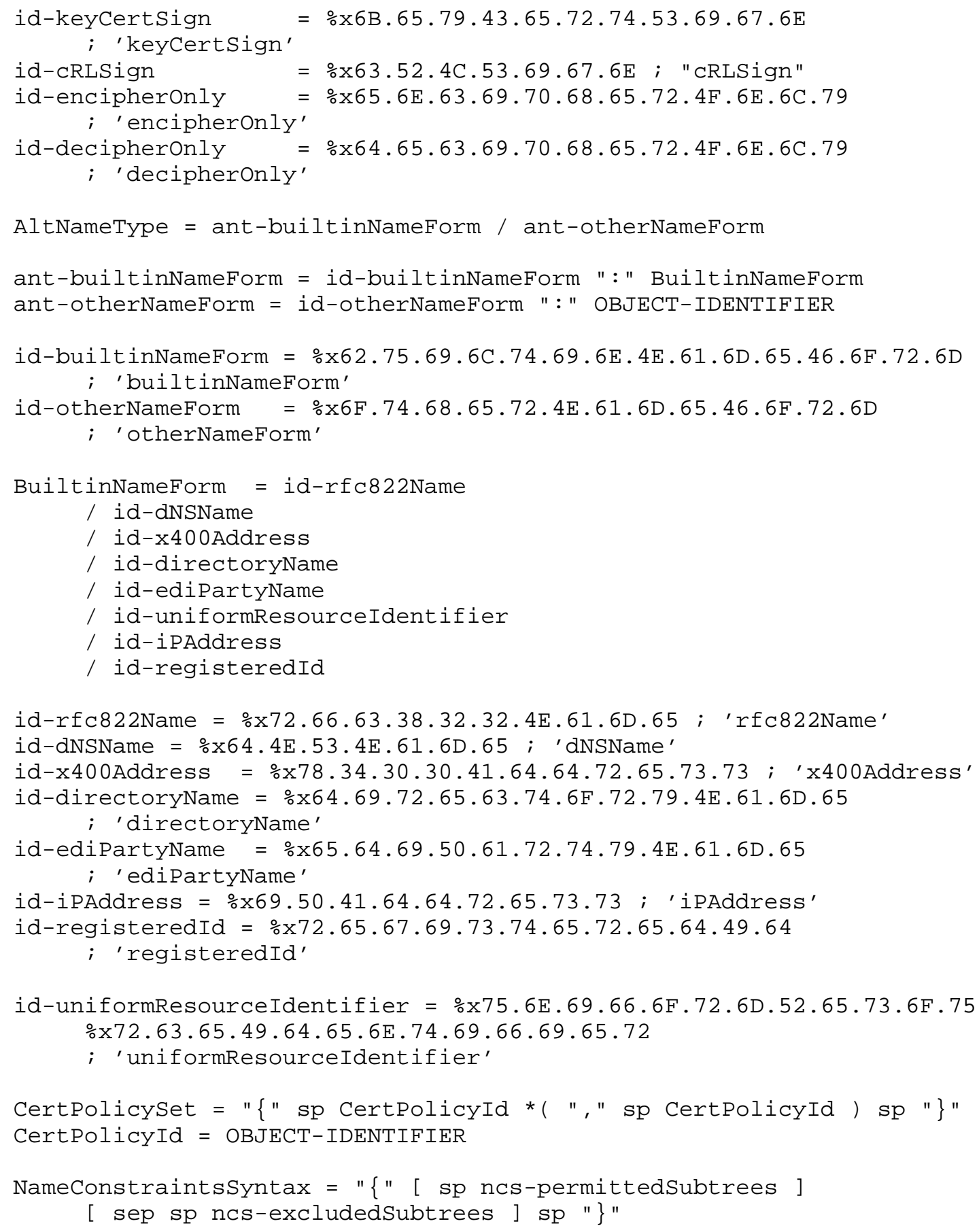




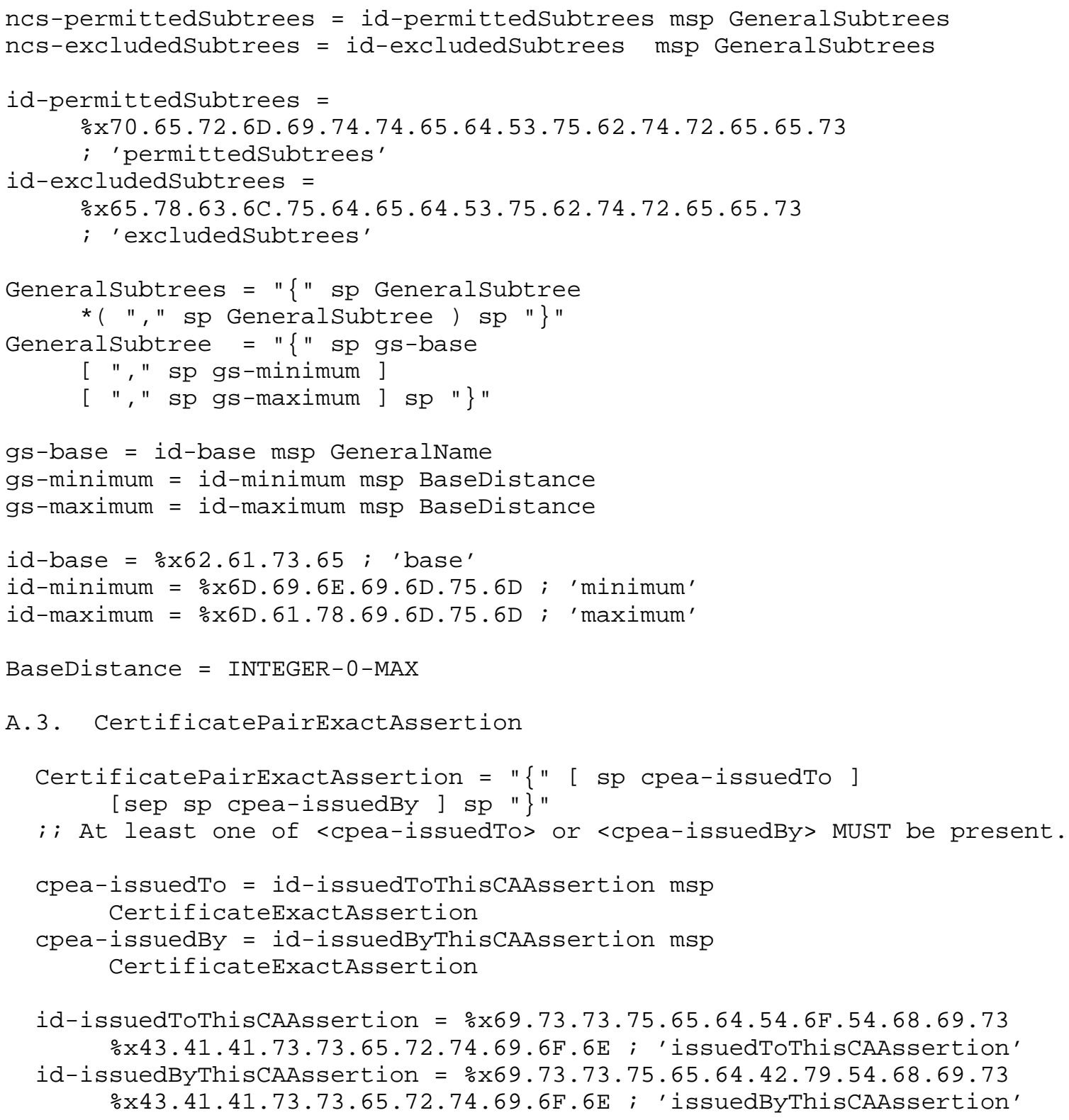




\section{A.4. CertificatePairAssertion}

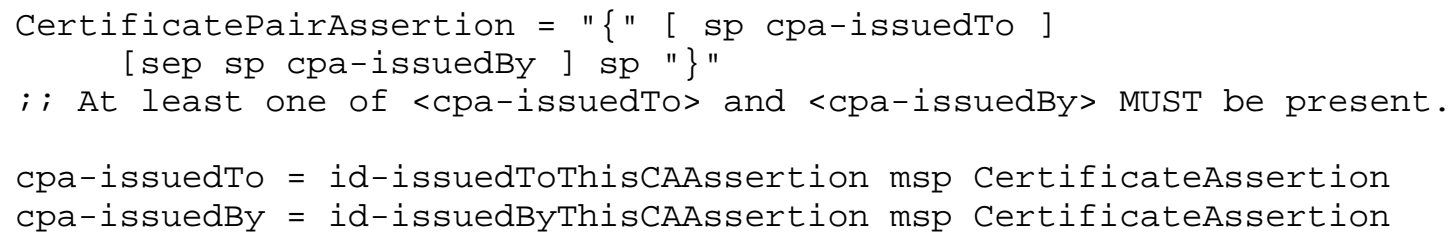

A.5. CertificateListExactAssertion

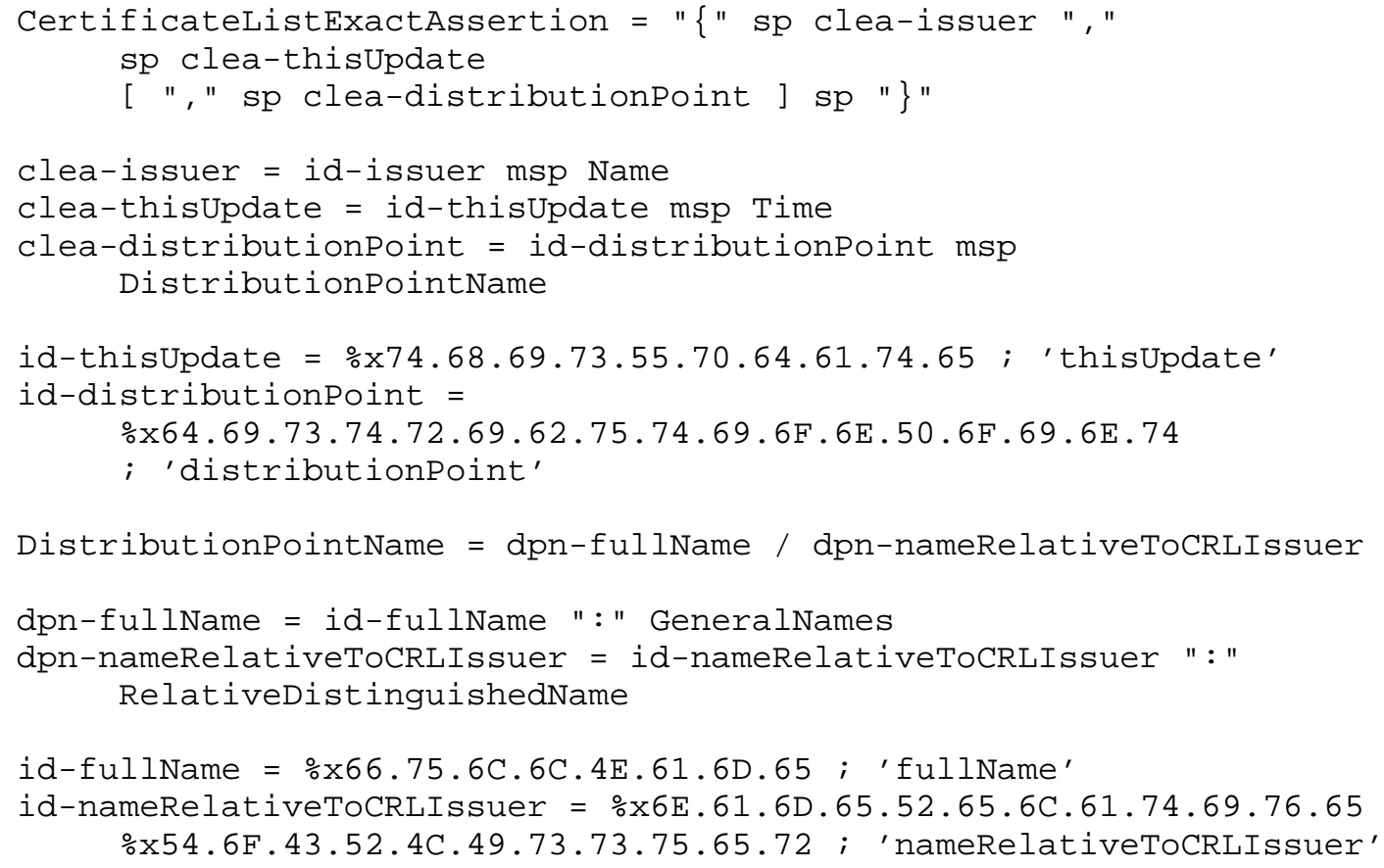




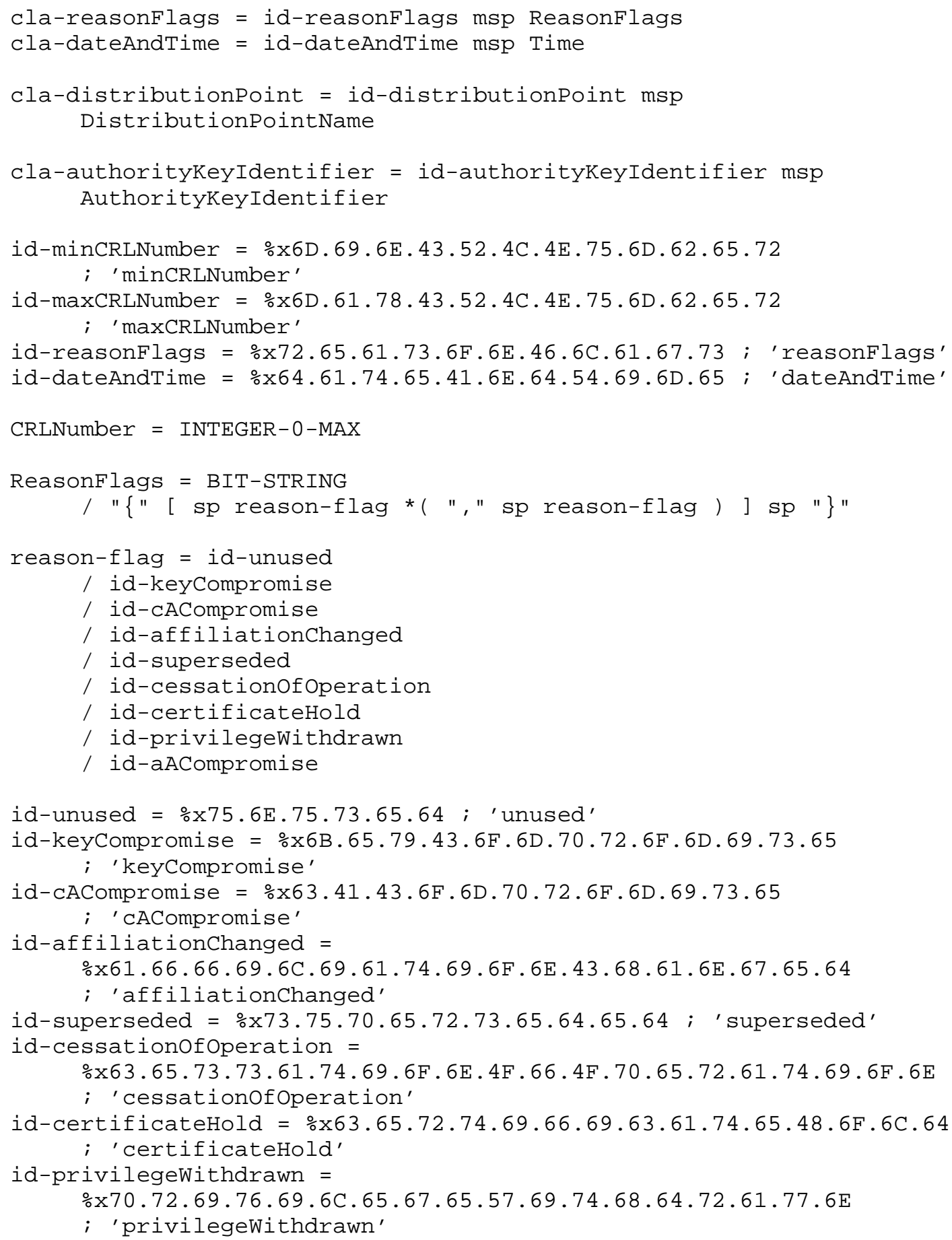




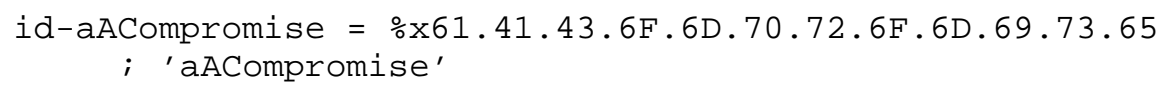

A.7. AlgorithmIdentifier

AlgorithmIdentifier $="\{"$ sp ai-algorithm

[ "," sp ai-parameters ] sp "\}"

ai-algorithm = id-algorithm msp OBJECT-IDENTIFIER

ai-parameters $=$ id-parameters msp Value

id-algorithm $=\frac{\circ}{2} 61.6 \mathrm{C} .67 .6 \mathrm{~F} .72 .69 .74 .68 .6 \mathrm{D} ;$ ' $^{\prime}$ algorithm'

id-parameters $=\frac{\circ}{0} 70.61 .72 .61 .6 \mathrm{D} .65 .74 .65 .72 .73 ;$ ' parameters'

Author's Address

Kurt D. Zeilenga

OpenLDAP Foundation

EMail: Kurt@openLDAP.org 
Full Copyright statement

Copyright (C) The Internet Society (2006).

This document is subject to the rights, licenses and restrictions contained in BCP 78, and except as set forth therein, the authors retain all their rights.

This document and the information contained herein are provided on an "AS IS" basis and THE CONTRIBUTOR, THE ORGANIZATION HE/SHE REPRESENTS OR IS SPONSORED BY (IF ANY), THE INTERNET SOCIETY AND THE INTERNET ENGINEERING TASK FORCE DISCLAIM ALL WARRANTIES, EXPRESS OR IMPLIED, INCLUDING BUT NOT LIMITED TO ANY WARRANTY THAT THE USE OF THE INFORMATION HEREIN WILL NOT INFRINGE ANY RIGHTS OR ANY IMPLIED WARRANTIES OF MERCHANTABILITY OR FITNESS FOR A PARTICULAR PURPOSE.

Intellectual Property

The IETF takes no position regarding the validity or scope of any Intellectual Property Rights or other rights that might be claimed to pertain to the implementation or use of the technology described in this document or the extent to which any license under such rights might or might not be available; nor does it represent that it has made any independent effort to identify any such rights. Information on the procedures with respect to rights in RFC documents can be found in BCP 78 and BCP 79 .

Copies of IPR disclosures made to the IETF Secretariat and any assurances of licenses to be made available, or the result of an attempt made to obtain a general license or permission for the use of such proprietary rights by implementers or users of this specification can be obtained from the IETF on-line IPR repository at http://www.ietf.org/ipr.

The IETF invites any interested party to bring to its attention any copyrights, patents or patent applications, or other proprietary rights that may cover technology that may be required to implement this standard. Please address the information to the IETF at ietf-ipreietf.org.

Acknowledgement

Funding for the RFC Editor function is provided by the IETF Administrative Support Activity (IASA). 